\title{
The measurement of muscle tone
}

\author{
E G Walsh MD FRCP FRSE
}

Department of Physiology, University of Edinburgh, EH8 9AG, Scotland.

Neurophysiologists have long been interested in phenomena associated with the activity of single nerve cells and much that has been found out has some implication for the management and understanding of spinal injuries. Others have been more concerned with integrative aspects, and it is with one aspect of this work that I am now concerned.

Key words: muscle tone; spinal injuries.

Much of Sherrington's classical work in neurophysiology dealt with muscle tone. The observations were on spinal and decerebrate cats and whilst much of the material remains valid other aspects now, some 50 or so years after his death at the age of 95 , are decidedly dated.

There have been many attempts to measure muscle tone. In one early method the rebound of a hammer was recorded, the theory being that the stiffer the muscle the quicker the rebound.

Popular and elementary, requiring only modest instrumentation is the Wartenberg test. The person is on a couch, the leg held horizontal and then allowed to drop. The swings are recorded. This method never seems to have been applied to joints other than the knee. In spasticity the fall is interrupted by a series of jerks, evidently due to the operation of phasic stretch reflexes. In Parkinsonism the leg may drop relatively slowly and, unlike a normal leg does not swing in a pendulous manner. In hypotonia, such as may be associated with lesions of the cerebellum, the pendulous oscillations, before the limb comes to rest, are increased.

Another method has been to drive the limb to and fro with power supplied by a geared motor and measure the resultant force between the instrument and the body. This would appear at first sight to be elegant but there are serious limitations. In moving a limb the resistance is not provided solely by the muscles but there are other forces and the method is in general bedevilled by the effects of inertia. The forces generated by inertia are proportional to the imparted acceleration, and for sinusoidal motion are thus related to the square of the frequency. In these instruments adjustment of the angle of motion is cumbersome. For large displacements the motion must be restricted to quite slow movements, so the effects of rapid stretching cannot be discerned. For rapid movement the motion must be quite small, but motion restricted to a few degrees will give only limited information. The investigators using this system have to make choices, neither of which are wholly satisfactory. Another system is to use, not sinusoidal, but constant velocity motion. Here the effects of inertia are restricted to the turning points where the direction of motion suddenly reverses; this system has only been used for slow movements. In spite of their widespread use these systems have proved to be very poor analytical tools.

Working with my collaborators, I have favoured a different system where it is not a certain velocity of movement which is defined by the instrument but a certain force, the motions of the unrestrained swings being recorded and analysed. The motion is in the horizontal plane to avoid the complications due to the influence of gravity. The apparatus as used at the wrist ${ }^{1}$ is shown in Figure 1. With motors in scale it is possible to make measurements at any of the accessible joints. Variants of the apparatus have been employed to investigate the biodynamics of the fingers, elbow, hip, knee and ankle, in normal subjects and in patients with a variety of neurological disorders including paraplegia. ${ }^{2-4}$ 


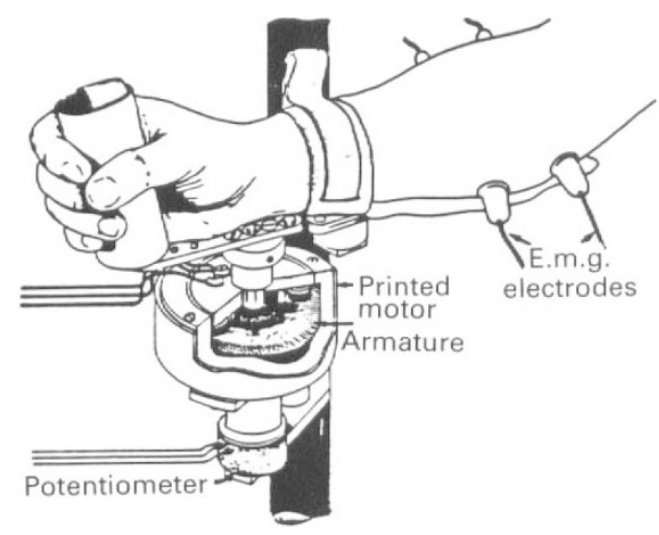

Figure 1 The hand is unconstrained as the motor is not geared, has almost no friction, and very little inertia. The force generated is proportional to the instantaneous current. The motion is recorded by the potentiometer and emg activity is picked up by surface electrodes.

When the apparatus is supplied with sinusoidal currents the frequency of which is varied, it is found that for a certain rate, the 'resonant frequency', the oscillations are largest. The resonant frequency (f) reflects muscle stiffness according to the equation:

$$
f=\frac{1}{2 \pi} \sqrt{\frac{K}{J}}
$$

where ' $\mathrm{J}$ ' is the inertia, and ' $\mathrm{K}$ ' stiffness. It follows that the stiffness of the muscle is related to the square of the resonant frequency.

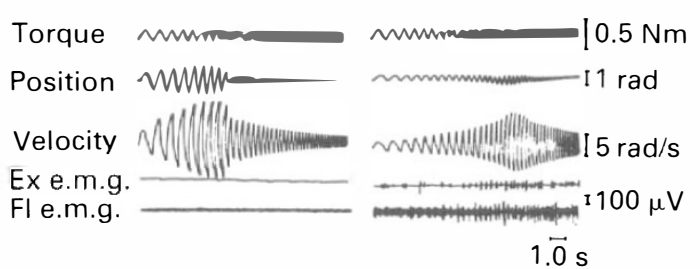

Figure 2 The normal limb (left) compared with the spastic side (right). The resonant frequency is clearly higher on the spastic side and there is emg activity modulated with the movements.

This system allows for observations at different torque levels to be made merely by adjusting a control. At low force levels limbs are in general stiffer than at higher levels. This is a reflection of thixotropy, referred to elsewhere in this journal (30(2): 113-115).

Resonance curves in a case of hemiplegia are shown in Figure 2. The resonant frequency is higher on the paretic side. ${ }^{5}$

Using the same apparatus other observations may rapidly be made. Thus if the waveform generator driving the system provides low frequency square waves the effect of abrupt stretching, and hence the presence or absence of phasic stretch reflexes may be ascertained.

In scientific work the method adopted is of fundamental importance. Anyone considering work on muscle tone should seriously consider the alternative methods available before embarking on any study.

\section{References}

1 Lakie M, Walsh EG, Wright GW (1984) Resonant frequency at the wrist demonstrated by the use of a torque motor: an instrumental analysis of muscle tone in man. J Physiol 353: 265-285.

2 Walsh EG (1976) Clonus: beats provoked by the application of a rhythmic force. J Neurol Neurosurg Psychiatry 39: 266-274.

3 Douglas AJ, Walsh EG, Wright GW, Edmond P (1989) Muscle tone around the human knee in paraplegia. Q J Exper Physiol 74: 897-905.

4 Douglas AJ, Walsh EG, Wright GW, Creasey GH, Edmond P (1991) The effects of neuromuscular stimulation on muscle tone at the knee in paraplegia. Exper Physiol 76: 367-367.

5 Lakie M, Walsh EG, Wright GW (1988) Assessment of human hemiplegic spasticity by a resonant frequency method. Clin Biomech 3: 173-178. 